\title{
Reachability in linear dynamical systems
}

\author{
Emmanuel Hainry \\ LORIA, Université Henri Poincaré \\ Campus scientifique, BP 239 - 54506 Vandøuvre-lès-Nancy, France \\ Emmanuel.Hainry@loria.fr
}

\begin{abstract}
Dynamical systems allow to modelize various phenomena or processes by only describing their local behaviour. It is however useful to understand the behaviour in a more global way. Checking the reachability of a point for example is a fundamental problem. In this document we will show that this problem that is undecidable in the general case is in fact decidable for a natural class of continuous-time dynamical systems: linear systems. For this, we will use results from the algebraic numbers theory such as GelfondSchneider's theorem.
\end{abstract}

Keywords: Dynamical Systems, Reachability, Skolem-Pisot problem, GelfondSchneider Theorem.

\section{Introduction}

A dynamical system is described by a function (the dynamics of the system) and a space on which this function is defined and in which the system will evolve. The evolution of a dynamical system is hence described in a very simple way but it can be hard to grasp where a point that undergoes the dynamics will go. Hence the problem of deciding whether given a certain point, the system will eventually reach another given point is fundamental.

Indeed, many natural phenomena can be described using dynamical systems. Examples come from mathematics [1], physics, biology [2]; the famous Lorenz' attractor [3] is an example of a dynamical system describing a meteorological phenomenon. However, as standard as those systems are, and as simple as the description of their dynamics may be, many important problems such as limit and reachability are undecidable.

Some positive results are known for some very specific classes but on the whole, it is very difficult to know much about such systems. Even considering polynomial systems yields many undecidable problems: [4] shows that it is possible to simulate a Turing machine using a polynomial dynamical system. It is hence undecidable whether or not a 
trajectory will reach the region corresponding to the halting state of the machine. This particular problem can be seen as a continuous version of the Skolem-Pisot problem [5, 6, 7] which studies whether a component of a discrete linear system will reach 0 . This problem is not different from deciding if this system reaches a hyperplane of the space, described by $y_{k}=0$ where $k$ is the number of the component considered. The SkolemPisot problem is equivalent to deciding whether a linear recurrent sequence reaches 0 .

It is still open whether the Skolem-Pisot problem is decidable. Some results are known but they don't yet enlighten the whole decision problem. As an example of recent developments, [7] shows that in small dimensions, the problem is decidable, and [8] shows that this problem is NP-hard. As this problem also arises in a continuous context it would be interesting to study the continuous Skolem-Pisot problem for continuoustime linear dynamical systems. Considering a continuous space may make the study of this problem easier than in a discrete space, indeed if two points on the two different sides of the aimed hyperplane are reached, continuity (and the intermediate values theorem) implies that the hyperplane will also be reached. Even if the discrete version of this problem had many possible interpretations, no natural interpretation appears in the continuous case.

The (point to point) reachability problem, which is undecidable in the general case, has been shown undecidable for various restricted classes of dynamical systems, such as Piecewise Constant Derivative systems [9] where the dynamics are really simple as it consists of a sharing of the space into regions where the derivative will be constant. Other results on the subject of reachability and undecidability of problems in hybrid systems are studied in [10, 11, 12, 13].

It has been shown [14] that in discrete-time linear dynamical systems, the reachability problem is decidable. The class of linear dynamical systems in the continuous field is hence a good candidate for a class of dynamical systems where reachability might be decidable. It is however not trivial to extend the result on discrete dynamical systems to continuous dynamical systems, indeed, it uses algebraic properties of the orbit that are not preserved in a continuous setting. In this paper, we will hence focus on linear continuous-time dynamical systems and show that reachability is decidable for those systems. This result is a necessary step if we want to study the continuous Skolem-Pisot problem that also deals with linear dynamical systems.

The section 2 presents the problems we are going and mathematical notions that will be useful in the following. The section 3 contains results of undecidability: for polynomial dynamical systems, the Skolem-Pisot problem and the reachability problem are undecidable. The next section is the core of this paper: it contains the theorem A which is the core of this paper and proves the decidability of reachability in linear dynamical systems. The proof of this result details in fact the algorithm used to decide the question. It is composed of two parts: the part 4.2 shows how to solve the problem in the specific case where the matrix is in Jordan form; the part 4.1 recalls that putting the matrix into Jordan form is doable. 


\section{Prerequisites}

\subsection{Linear continuous-time dynamical systems}

The dynamics of a linear dynamical system are described by a linear differential equation. To describe such a system, we take a matrix of real numbers which will represent the dynamics and a vector of reals that is the initial point. We use here classical definitions and notations that can be found in [15].

Definition 1 (Linear continuous-time dynamical system) Given a matrix $A \in$ $\mathbb{R}^{n \times n}$ and a vector $X_{0} \in \mathbb{R}^{n}$. We define $X$ as the solution of the following Cauchy problem:

$$
\begin{cases}X^{\prime} & =A X \\ X(0) & =X_{0} .\end{cases}
$$

$X$ is called a trajectory of the system.

Definition 2 (Reachability) Given $A \in \mathbb{R}^{n \times n}, X_{0} \in \mathbb{R}^{n}, Y \in \mathbb{R}^{n}$, the system is said to reach $Y$ from $X_{0}$ if there exists $t \in \mathbb{R}$ such that $X(t)=Y$ with $X$ the trajectory defined with the dynamics $A$ and the initial point $X_{0}$.

Definition 3 ( $\omega$-limit points) Given a trajectory $X$, a point $Y$ is an $\omega$-limit point of $X$ if there is an diverging increasing sequence $\left(t_{n}\right) \in \mathbb{R}^{\mathbb{N}}$ such that $Y=\lim _{n \rightarrow+\infty} X\left(t_{n}\right)$.

Definition 4 ( $\omega$-limit sets) The $\omega$-limit set of a dynamical system is the set of its $\omega$-limit points: $\omega(X)=\cap_{n} \overline{\cup_{t>n} X(t)}$, where $\bar{A}$ is the closure of the set $A$.

The problems we are interested in are the reacability problem (which we will prove decidable in Linear Dynamical Systems) and the Skolem-Pisot problem.

Problem 1 (Reachability problem) Given a trajectory $X$ defined from $A \in \mathbb{K}^{n \times n}$ and $X_{0} \in \mathbb{K}^{n}$, a point $Y \in \mathbb{K}^{n}$, decide whether $Y$ can be reached from $X_{0}$.

The classical Skolem-Pisot problem originally consists in determining if a linear recurrent sequence has a zero. It can however be defined as a hyperplane reachability problem.

Problem 2 (Skolem-Pisot problem) Given a trajectory $X$, given $C \in \mathbb{K}^{n}$ defining an hyperplane ${ }^{1}$ of $\mathbb{K}^{n}$, decide if $\exists t \in \mathbb{R}$ such that $C^{T} X(t)=0$ ? In other words, does the trajectory $X$ intersect the hyperplane defined by $C$ ?

The problems we will consider will be those for which the field $\mathbb{K}$ is in fact the set of rational numbers $\mathbb{Q}$.

\footnotetext{
${ }^{1}$ The hyperplane defined by $C$ is the set of points $Y$ such that $C^{T} Y=[0]$
} 


\subsection{Polynomials}

Let us now recall a few notations, mathematical tools and algorithms on polynomials. In the following, we use a field $\mathbb{K}$ that is a subfield of $\mathbb{C}$.

Definition 5 (Ring of polynomials) We denote $\mathbb{K}[X]$ the ring of one variable polynomials with coefficients in $\mathbb{K}$. A polynomial can be written as $P(X)=\sum_{i=1}^{n} a_{i} X^{i}$, with $a_{i} \in \mathbb{K}$ and $a_{n} \neq 0$. The integer $n$ is the degree of $P$.

Definition 6 (Roots of a polynomial) The set $Z(P)$ of roots of a polynomial $P$ is defined as $Z(P)=\{x \in \mathbb{C} ; P(x)=0\}$

Definition 7 (Algebraic numbers) The set of roots of polynomials with coefficients in $\mathbb{Q}$ is the set of algebraic numbers.

An algebraic number can be represented uniquely by the minimal polynomial it nulls (minimal in $\mathbb{Q}[X]$ for the division) and a ball containing only one root of the polynomial. Note that the size of the ball can be chosen using only the values of the coefficients of the polynomial as [16] shows a bound on the distance between roots of a polynomial from its coefficient.

Definition 8 (Representation of an algebraic number) An algebraic number $\alpha$ will be represented by $(P,(a, b), \rho)$ where $P$ is the minimal polynomial of $\alpha, a+\mathrm{i} b$ is an approximation of $\alpha$ such that $|\alpha-(a+\mathrm{i} b)|<\rho$ and $\alpha$ is the only root of $P$ in the open ball $\mathcal{B}(a+\mathrm{i} b, \rho)$.

It can be shown that given the representations of two algebraic numbers $\alpha$ and $\beta$, the representations of $\alpha+\beta, \alpha-\beta, \alpha \beta$ and $\alpha / \beta$ can be computed. See [17, 18] for details.

We will also need specific results on algebraic numbers that come from 19, 20].

Proposition 1 (Baker) Given $\alpha \in \mathbb{C}-\{0\}, \alpha$ and $\mathrm{e}^{\alpha}$ are not both algebraic numbers.

Theorem 1 (Gelfond-Schneider) Let $\alpha$ and $\beta$ be two algebraic numbers. If $\alpha \notin$ $\{0,1\}$ and $\beta \notin \mathbb{Q}$, then $\alpha^{\beta}$ is not algebraic

\subsection{Matrices}

Definition 9 (Characteristic polynomial) Given a matrix $A \in \mathbb{K}^{n \times n}$, its characteristic polynomial is $\chi_{A}(X)=\operatorname{det}\left(X I_{n}-A\right)$

Definition 10 (Exponential of a matrix) Given a matrix A, its exponential denoted $\exp (A)$ is the matrix

$$
\sum_{i=1}^{+\infty} \frac{1}{i !} A^{i}
$$


Note that the exponential is well defined for all real matrices.

All matrices can be put in Jordan form, which allows to compute easily the exponential. To find more about Jordan matrices and blocks, the reader may consult [15] or [21.

Definition 11 (Jordan block) A Jordan block is a square matrix of one of the two following forms

$$
\left[\begin{array}{cccc}
\lambda & & & \\
1 & \lambda & & \\
& \ddots & \ddots & \\
& & 1 & \lambda
\end{array}\right] ; \quad\left[\begin{array}{cccc}
B & & & \\
I_{2} & B & & \\
& \ddots & \ddots & \\
& & I_{2} & B
\end{array}\right] \text { with } B=\left[\begin{array}{cc}
a & -b \\
b & a
\end{array}\right] \text { and } I_{2}=\left[\begin{array}{cc}
1 & 0 \\
0 & 1
\end{array}\right]
$$

Definition 12 (Jordan form) A matrix that contains Jordan blocks on its diagonal is said to be in Jordan form.

$$
\left[\begin{array}{cccc}
D_{1} & 0 & \cdots & 0 \\
0 & D_{2} & \ddots & \vdots \\
\vdots & \ddots & \ddots & 0 \\
0 & \cdots & 0 & D_{n}
\end{array}\right]
$$

Proposition 2 ([21]) Any matrix $A \in \mathbb{R}^{n \times n}$ is similar to a matrix in Jordan form. In other words,

$$
\exists P \in G L\left(\mathbb{R}^{n \times n}\right) \text { and } J \text { in Jordan form such that } A=P^{-1} J P .
$$

\section{Undecidability for polynomial dynamical systems}

Many biological phenomena can be modelised using polynomial dynamical systems rather than linear dynamical systems. A famous example comes from meteorological systems which were described by Lorenz in [3]. Lorenz' attractor has a quite chaotic behaviour which gives the intuition that the reachability problem in polynomial dynamical systems is not decidable. Other polynomial differential systems yields fractal basins of attraction. In other words, this dynamical systems has exactly two $\omega$-limit points depending on the initial point and, the set of starting points that will lead to the first of those attractors is a fractal, for example a Julia set.

In those systems, from already known results, we can infer that the Skolem-Pisot problem and the reachability problem are undecidable.

Theorem 2 The Skolem-Pisot problem is undecidable for polynomial dynamical systems.

Proof: From [4], we know that it is possible to simulate a Turing machine using a polynomial differential system. The halt of the Turing machine is then equivalent to the system reaching the hyperplane $z=q_{f}$ which stands for the halting state. This is an instance of the Skolem-Pisot problem. 
Theorem 3 Reachability is undecidable for polynomial dynamical systems.

Proof: Let us modify the Turing machine of the previous proof so that from the halting state, the machine erases its tape then enters a special state. Simulating this machine by the same mechanism from [4], the dynamical system reaches the point representing blank tapes and special state if and only if the original machine halts. This means we can translate any instance of the halting problem into a reachability in polynomial differential systems problem.

\section{Decidability for linear dynamical systems}

This section is devoted to proving the main theorem of this article: theorem 4 .

Theorem 4 The reachability problem for continuous time linear dynamical systems with rational coefficients is decidable.

To decide whether a point is reachable we will try to obtain an expression of the trajectory $X$ that is usable and with this expression search for the different $t$ that could be solution. We will first consider the case where the matrix is in Jordan form: this case will be studied in section 4.2. The section 4.1 will show how to put the matrix in Jordan form. Note that the Jordan matrix will have algebraic coefficients and not only rational ones.

\subsection{To put the matrix in Jordan form}

To be able to do what we have done in the previous section, we will want to find a Jordan matrix similar to the one considered. Building the Jordan form of a matrix implies knowing its eigenvalues, for that we need to compute the roots of the characteristic polynomial of the matrix.

This consist in the following steps that are classical: computing the characteristic polynomial; factorizing the polynomial in $\mathbb{Q}[X]$; computing the roots; jordanizing the matrix.

\subsection{If the matrix is in Jordan form}

Let us suppose that the matrix $A$ is in Jordan form with algebraic coefficients and that the $X_{0}$ and $Y$ vectors are also composed of algebraic elements. This means $A=$ $\left[\begin{array}{cccc}D_{1} & 0 & \cdots & 0 \\ 0 & D_{2} & \ddots & \vdots \\ \vdots & \ddots & \ddots & 0 \\ 0 & \cdots & 0 & D_{k}\end{array}\right]$ with the $D_{i}$ being Jordan blocks.

The solution of the Cauchy system $\left\{\begin{array}{l}X^{\prime}=A X \\ X(0)=X_{0}\end{array}\right.$ is $X(t)=\exp (t A) X_{0}$. 
We then need to compute the exponential of $t A$. It is easy to check that

$$
\exp (t A)=\left[\begin{array}{llll}
\exp \left(t D_{1}\right) & & & \\
& \exp \left(t D_{2}\right) & & \\
& & \ddots & \\
& & & \exp \left(t D_{k}\right)
\end{array}\right]
$$

Finding a $t \in \mathbb{R}$ such that $X(t)=Y$ is equivalent to finding such a $t$ for each component $i$ and ensuring this is always the same $t$. We are going to solve the equation Jordan block by Jordan block. It means we choose an $i$ such that the corresponding part of $X_{0}$ is not null (in the other case it is easy to decide if either all $t \in \mathbb{R}$ will be solutions or no $t$ will be solution) and search for a $t$ such that $\exp \left(t D_{i}\right)\left[\begin{array}{c}x_{1} \\ \vdots \\ x_{n_{i}}\end{array}\right]=\left[\begin{array}{c}y_{1} \\ \vdots \\ y_{n_{i}}\end{array}\right]$ where the $x_{j}$ and $y_{j}$ are the elements of $X_{0}$ and $Y$ corresponding to the block $i$. To simplify the notations, we will forget $i$ and just consider the problem as being $\exp (t D) X_{0}=Y$ and $k$ being the size of this block.

There are two cases to consider: the two different forms of Jordan blocks. For each of those cases, a few sub cases are to be considered which revolve around the nullity of the real part of the eigenvalue. Let us note that as we deal with algebraic numbers, it is possible to verify if the real part or the imaginary part is null.

\subsubsection{First form: a real eigenvalue}

The first form of Jordan blocks corresponds to a real eigenvalue $\lambda$. Two cases need to be dealt with: $\lambda=0$ and $\lambda \neq 0$

If $\lambda \neq 0$. The exponential is $\exp (t D)=\mathrm{e}^{t \lambda}\left[\begin{array}{ccccc}1 & & & & \\ t & 1 & & & \\ \frac{t^{2}}{2} & t & 1 & & \\ \vdots & \ddots & \ddots & \ddots & \\ \frac{t^{k}}{k !} & \cdots & \frac{t^{2}}{2} & t & 1\end{array}\right]$. If $X_{0_{1}}$ is not 0 , then there is at most one $t \in \mathbb{R}$ solution. Indeed, let us consider $x_{i}$, the first non null element of $\left\{x_{1}, x_{k}\right\}$. The only possible $t$ is then $\frac{1}{\lambda} \ln \left(\frac{y_{i}}{x_{i}}\right)$.

We want to verify that this $t$ is coherent with the rest of the block. Let us remark that $\mathrm{e}^{t \lambda}=\frac{y_{i}}{x_{i}}$ is an algebraic number. If the block has size more than 1 , then $t$ verifies some algebraic equations hence the proposition 1 says $\lambda t=0$, it is easy to verify if $t=0$ is the solution of the block. 
If $\lambda=0$. The case with $\lambda=0$ means we are searching for a $t$ such that

$$
\left[\begin{array}{ccccc}
1 & & & \\
t & 1 & & \\
t^{2} / 2 & t & 1 & \\
\vdots & \ddots & \ddots & \ddots & \\
t^{k} & \cdots & t^{2} / 2 & t & 1
\end{array}\right]\left[\begin{array}{c}
x_{1} \\
\vdots \\
x_{k}
\end{array}\right]=\left[\begin{array}{c}
y_{1} \\
\vdots \\
y_{k}
\end{array}\right]
$$

For such a $t$ to exist, we need to have $x_{1}=y_{1}, x_{2}+t x_{1}=y_{2}, \ldots$ Let us say that $x_{i}$ is the first non-null element of $X$. Then the only candidate for $t$ is $\frac{y_{i+1}-x_{i+1}}{x_{i}}$. Since this candidate is algebraic, it is easy to check whether this $t$ is a solution for the block.

\subsubsection{Second form}

The second form corresponds to complex eigenvalues. The Jordan block is

$$
D=\left[\begin{array}{cccc}
B & & & \\
I_{2} & B & & \\
& \ddots & \ddots & \\
& & I_{2} & B
\end{array}\right] \text { with } B=\left[\begin{array}{cc}
a & -b \\
b & a
\end{array}\right] \text { and } I_{2}=\left[\begin{array}{ll}
1 & 0 \\
0 & 1
\end{array}\right] .
$$

The exponential is

$$
\exp (D)=\mathrm{e}^{t a}\left[\begin{array}{ccccc}
B_{2} & & & & \\
t B_{2} & B_{2} & & & \\
\frac{t^{2}}{2} B_{2}^{2} & t B_{2} & B_{2} & & \\
\vdots & \ddots & \ddots & \ddots & \\
\frac{t^{k}}{k !} B_{2}^{k} & \cdots & \frac{t^{2}}{2} B_{2}^{2} & t B_{2} & B_{2}
\end{array}\right] \text { with } B_{2}=\left[\begin{array}{cc}
\cos (t b) & -\sin (t b) \\
\sin (t b) & \cos (t b)
\end{array}\right] .
$$

There are two cases to consider, whether $a$ is null or not.

If $a=0$. In the case where the eigenvalue has a null real part, the $\exp (t a)$ term disappears. Let us suppose $c$ is the smallest odd number such that $x_{j} \neq 0$ or $x_{j+1} \neq 0$. We first want to solve $\left[\begin{array}{c}y_{j} \\ y_{j+1}\end{array}\right]=B_{2}\left[\begin{array}{c}x_{j} \\ x_{j+1}\end{array}\right]$. Let us remark that, since $B_{2}$ is a rotation, if $\sqrt{x_{j}^{2}+x_{j+1}^{2}} \neq \sqrt{y_{j}^{2}+y_{j+1}^{2}}$, there is no solution and in the other case, there is an infinity of solutions. We can express the solution of this system $t \in \alpha+\frac{2 \pi}{b} \mathbb{Z}$ where $\alpha$ is not explicitly algebraic as its expression uses $\tan ^{-1}$. Let us remark that for all those candidate $t$, the matrix $B_{2}$ is the same, namely $B_{2}=\left[\begin{array}{cc}\cos (\alpha) & -\sin (\alpha) \\ \sin (\alpha) & \cos (\alpha)\end{array}\right]$. Those $\cos (\alpha)$ and $\sin (\alpha)$ are algebraic numbers that can be computed: we can write an expression in $x_{j}, x_{j+1}, y_{j}$ and $y_{j+1}$ for each combination of signs for those numbers. ${ }^{2}$

\footnotetext{
${ }^{2}$ For example, if $x_{j}>0, x_{j+1}>0, y_{j}>0$ and $y_{j+1}>0$, we have $\sin (\alpha)=\sqrt{\frac{y_{j}^{2}}{y_{j}^{2}+y_{j+1}^{2}} \frac{x_{j}^{2}+x_{j+1}^{2}}{\left(x_{j}+x_{j+1}\right)^{2}}}$ and $\cos (\alpha)$ satisfies a similar expression.
} 
We then have to verify whether the following components of $X$ and $Y$ are compatible with those $t$. We have $\left[\begin{array}{l}y_{j+2} \\ y_{j+3}\end{array}\right]=t\left[\begin{array}{c}y_{j} \\ y_{j+1}\end{array}\right]+\left[\begin{array}{cc}\cos (\alpha) & -\sin (\alpha) \\ \sin (\alpha) & \cos (\alpha)\end{array}\right]\left[\begin{array}{l}x_{j+2} \\ x_{j+3}\end{array}\right]$. Since $y_{j}$ or $y_{j+1}$ is non null (as $\sqrt{y_{j}^{2}+y_{j+1}^{2}}=\sqrt{x_{j}^{2}+x_{j+1}^{2}} \neq 0$ ), there is then at most one solution and we can express it as an algebraic number.

Conclusion for $a=0$. We are able to discriminate 3 possible cases: either there is no solution, either there is exactly one candidate $t$ (defined with a fraction and a few subtractions of elements of $X$ and $Y$ ) either there is an infinity of candidate $t$ (defined as $\pm \alpha+\frac{2 \pi}{b} \mathbb{Z}$ with the $\alpha$ being fractions of elements of $X$ and $Y$ ). This last case will need to be compared with the results for the other Jordan blocks to decide whether there will be solutions or not for the whole system.

If $a \neq 0 . \quad$ In the case where $a \neq 0$, the term $\exp (t a)$ makes the solution not simply turn around the origin but describe a spiral. If $a>0$, this spiral is diverging, if $a<0$ it is converging to the origin. We just have to study the norm of $Y$.

We want to solve the system $\mathrm{e}^{t a}\left[\begin{array}{cc}\cos (t b) & -\sin (t b) \\ \sin (t b) & \cos (t b)\end{array}\right]\left[\begin{array}{l}x_{1} \\ x_{2}\end{array}\right]=\left[\begin{array}{l}y_{1} \\ y_{2}\end{array}\right]$ with $x_{1}$ or $x_{2}$ not null (if they are, we will choose another $x_{j}$ ). Let us consider the norms of the two sides of this equation: $\mathrm{e}^{t a} \sqrt{x_{1}^{2}+x_{2}^{2}}=\sqrt{y_{1}^{2}+y_{2}^{2}}$. As we have chosen $x_{1}$ or $x_{2}$ to be non null, we can write $\mathrm{e}^{t a}=\sqrt{\frac{y_{1}^{2}+y_{2}^{2}}{x_{1}^{2}+x_{2}^{2}}}$. We hence have exactly one $t$ candidate to be the solution. This $t$ is the logarithm of an algebraic number and we can check whether $t b$ is the correct angle (this is the combination of a non algebraic solution with an infinity of solutions).

Putting together the solutions. As we have seen, for one block, we may have no solution, one solution or an infinity of solutions. We must then bring the blocks together. In the case where one block has no solution, the problem is solved. In the case where there is exactly one solution, it can be algebraic (if $\lambda=0$, or $\lambda>0$ and there is more than one component to check), in which case it is easy to compute formally $\exp (t A) X_{0}$ and compare it with $Y$.

If we only have non explicitly algebraic solutions, we know that the solution must verify $\forall i, \exp \left(a_{i} t\right)=z_{i}$ with $a_{i}$ and $z_{i}$ algebraic numbers. We must then have $\mathrm{e}^{\frac{a_{1}}{a_{2}} \ln \left(z_{1}\right)}=z_{2}$. From theorem $\mathbb{1}$, it implies that $a_{1} / a_{2} \in \mathbb{Q}$ or $z_{1} \in\{0,1\} . z_{1}=0$ is not compatible, $z_{1}=1$ means that $t$ is rational and does not belong to this case. $a_{1} / a_{2} \in \mathbb{Q}$ can be checked easily (it means the degree of the minimal polynomial is at most 1 ). Then we must check that $z_{1}^{a_{1} / a_{2}}=z_{2}$ which is possible for a rational exponent. This verification must be done for all pairs of $a_{i}$.

If we have several infinities of candidates, we have to decide whether those infinities have a common point. To decide whether the $\alpha_{i}+\frac{2 \pi}{b_{i}} \mathbb{Z}$ intersect, we need to know whether the $b_{i}$ have an integer common multiple. If they don't, then there will exist an infinity of $t$ belonging to all those sets; if they do, only a finite number of $t$ need to be tested.

The last case is if we have on one hand a non algebraic solution and on the other hand an infinity of solutions. We can summarize this case as the simultaneous resolution of 
two constraints: $\left\{\begin{array}{l}\mathrm{e}^{a t}=z \\ {\left[\begin{array}{cc}\cos (b t) & -\sin (b t) \\ \sin (b t) & \cos (b t)\end{array}\right]\left[\begin{array}{l}x_{1} \\ x_{2}\end{array}\right]=\left[\begin{array}{l}y_{1} \\ y_{2}\end{array}\right] \text {. We will rephrase the second part }}\end{array}\right.$ as $\left[\begin{array}{cc}\mathrm{i} b t & 0 \\ 0 & \mathrm{e}^{-\mathrm{i} b t}\end{array}\right]\left[\begin{array}{cc}1 & -\mathrm{i} \\ 1 & \mathrm{i}\end{array}\right]\left[\begin{array}{l}x_{1} \\ x_{2}\end{array}\right]=\left[\begin{array}{cc}1 & -\mathrm{i} \\ 1 & \mathrm{i}\end{array}\right]\left[\begin{array}{l}y_{1} \\ y_{2}\end{array}\right]$.

And we can write the whole system as the following: $\left\{\begin{array}{l}\mathrm{e}^{a t}=z \\ \mathrm{e}^{\mathrm{i} b t}=z_{2} \\ \mathrm{e}^{-\mathrm{i} b t}=z_{3}\end{array}\right.$, where $a, b, z$, $z_{2}$, and $z_{3}$ are algebraic numbers (some are complex). We have already been confronted with such a system (but it had only two components) and we know that from theorem 1 it means that $\mathrm{i} \frac{b}{a}$ belongs to $\mathbb{Q}$ or $z \in\{0,1\}$. i $\frac{b}{a} \in \mathbb{Q}$ can be verified easily as it is an algebraic number; $z=0$ is impossible, 1 means $\mathrm{e}^{a t}=1$ hence $a=0$ (which belongs to another case) or $t=0$ hence $z_{2}=z_{3}=1$ in which case, $t=0$ is a solution to the problem.

\section{References}

[1] Hirsch, M.W., Smale, S., Devaney, R.: Differential Equations, Dynamical Systems, and an Introduction to Chaos. Elsevier Academic Press (2003)

[2] Murray, J.D.: Mathematical Biology. Second edn. Volume 19 of Biomathematics. Springer Verlag, Berlin, Germany (1993)

[3] Lorenz, E.N.: Deterministic non-periodic flow. Journal of the Atmospheric Sciences 20 (1963) 130-141

[4] Graça, D.S., Campagnolo, M.L., Buescu, J.: Robust simulations of Turing machines with analytic maps and flows. In Cooper, S.B., Löwe, B., Torenvliet, L., eds.: CiE 2005. Volume 3526 of LNCS., Springer (2005) 169-179

[5] Mignotte, M.: Suites récurrentes linéaires. In: Séminaire Delange-Pisot-Poitou. Théorie des nombres. Volume 15. (1974) G14-1-G14-9

[6] Berstel, J., Mignotte, M.: Deux propriétés décidables des suites récurrentes linéaires. Bulletin de la Société Mathématique de France 104 (1976) 175-184

[7] Halava, V., Harju, T., Hirvensalo, M., Karhumäki, J.: Skolem's problem - on the border between decidability and undecidability. Technical Report 683, Turku Center for Computer Science (2005)

[8] Blondel, V., Portier, N.: The presence of a zero in an integer linear recurrent sequence is NP-hard to decide. Linear algebra and its Applications 351-352 (2002) $91-98$

[9] Bournez, O.: Complexité algorithmique des systèmes dynamiques continus et hybrides. PhD thesis, École Normale Supérieure de Lyon (1999) 
[10] Asarin, E., Maler, O., Pnueli, A.: Reachability analysis of dynamical systems having piecewise-constant derivatives. Theoretical Computer Science 138 (1995) 35-65

[11] Asarin, E., Schneider, G.: Widening the boundary between decidable and undecidable hybrid systems. In Brim, L., Jancar, P., Kretínský, M., Kucera, A., eds.: CONCUR 2002. Volume 2421 of LNCS., Springer (2002) 193-208

[12] Asarin, E., Schneider, G., Yovine, S.: On the decidability of the reachability problem for planar differential inclusions. In Benedetto, M.D.D., Sangiovanni-Vincentelli, A.L., eds.: HSCC 2001. Volume 2034 of LNCS., Springer (2001) 89-104

[13] Blondel, V., Tsitsiklis, J.N.: A survey of computational complexity results in systems and control. Automatica 36(9) (2000) 1249-1274

[14] Kannan, R., Lipton, R.J.: Polynomial-time algorithm for the orbit problem. Journal of the ACM 33(4) (1986) 808-821

[15] Hirsch, M.W., Smale, S.: Differential Equations, Dynamical Systems, and Linear Algebra. Academic Press (1974)

[16] Mignotte, M.: An inequality about factors of polynomials. Mathematics of Computation 28(128) (1974) 1153-1157

[17] Bostan, A.: Algorithmique efficace pour des opérations de base en calcul formel. $\mathrm{PhD}$ thesis, École polytechnique (2003)

[18] Brawley, J.V., Carlitz, L.: Irreducibles and the composed product for polynomials over a finite field. Discrete Mathematics 65(2) (1987) 115-139

[19] Baker, A.: Transcendental Number Theory. Cambridge University Press (1990)

[20] Gelfond, A.O.: Transcendental and Algebraic Numbers. Dover Publications (2003)

[21] Lelong-Ferrand, J., Arnaudiès, J.M.: Cours de mathématiques, tome 1 : algèbre. Dunod (1971)

[22] von zur Gathen, J., Gerhard, J.: Modern Computer Algebra. Cambridge University Press (2003)

[23] Cohen, H.: A Course in Computational Algebraic Number Theory. Springer (1993) 


\section{A. Appendix}

\section{A.1. Factorizing a polynomial in $\mathbb{Q}[X]$}

The characteristic polynomial $\chi_{A}(X)$ of the matrix $A \in \mathbb{Q}^{n \times n}$ belongs to $\mathbb{Q}[X]$. We will first factorize $\chi_{A}(X)$ in $\mathbb{Q}[X]$ to obtain some square-free polynomials. This is a classical problem. One solution is to use Yun's algorithm [22, p. 371] that writes our polynomial $\chi_{A}$ into the form

$$
\chi_{A}=\prod_{i} R_{i}^{i}
$$

where the $R_{i}$ are square-free and do not share roots. The polynomial $\prod R_{i}$ is then a square-free polynomial that has the same roots as $P$.

Proposition 3 Suppose given a polynomial $P$ that we can write as

$$
P=\prod\left(X-\alpha_{j}\right)^{\beta_{j}}
$$

with the $\alpha_{j}$ distinct. Let $Q=P / \operatorname{gcd}\left(P, P^{\prime}\right)$, then $Q$ is square-free and

$$
Q=\prod\left(X-\alpha_{j}\right) .
$$

We then want to factorize this polynomial $Q$ in irreducible factors in $\mathbb{Q}[X]$. This problem is again a classical problem. An algorithm that achieves this goal is for example presented in [23, p. 139].

Proposition 4 Given a square-free polynomial $P \in \mathbb{Q}[X]$, we can compute its factorization in $\mathbb{Q}[X]$.

So we have obtained $Q=\prod Q_{i}$ with the $Q_{i}$ being polynomial that are irreducible in $\mathbb{Q}[X]$

\section{A.2. Computing the roots}

To obtain $\chi_{A}$ 's roots, we are going to compute the roots of $Q$. Those are algebraic numbers. We only then need to compute a representation of each of those roots. It means finding the minimal polynomial and giving a rational approximation of the root and an error bound to discriminate other roots of the minimal polynomial. Let us consider a $Q_{i}$.

There can be both real roots and complex roots that are not real. Sturm's theorem allows us to know the number of each of them [23, pp. 153-154]. We can then find the real roots with, for example, Newton's iteration algorithm [22, sec. 9.4]. The complex roots will for example be computed with Schönhage's method.

From this, we obtain approximations of the roots of the polynomial $Q_{i}$. Let $\alpha_{j}$ be one of those roots. The minimal polynomial of $\alpha_{j}$ divides $Q_{i}$ and belongs to $\mathbb{Q}[X]$. As $Q_{i}$ is irreducible in $\mathbb{Q}[X]$, the minimal polynomial can only be $Q_{i}$ (1 has no root and hence cannot be a minimal polynomial).

We then obtain a factorization of $Q$ as $\prod\left(X-\alpha_{j}\right)$ with the $\alpha_{j}$ explicitly defined as algebraic numbers. 


\section{A.3. Jordanizing the matrix}

The final step to be able to use the method described earlier is to do the factorization of $\chi_{A}$ in $\mathbb{C}[X]$. In fact, it is sufficient to do it in $\mathbb{Q}\left(\left\{\alpha_{j}\right\}\right)[X]$ to obtain a factorization into monomials. So from now on, we will work in $\mathbb{Q}\left(\alpha_{j}\right)$ which is the field generated from $\mathbb{Q}$ and the algebraic numbers $\left\{\alpha_{j}\right\}$.

To find the multiplicity of each root, we just need to know how many times the minimal polynomial divides $\chi_{A}$. We then obtain a decomposition

$$
\chi_{A}(X)=\prod\left(X-a_{i}\right)^{b_{i}} \prod\left(\left(X-\alpha_{i}\right)\left(X-\bar{\alpha}_{i}\right)\right)^{\beta_{i}}
$$

with the $\alpha_{i}$ being the complex not real roots and the $a_{i}$ the real roots.

The different Jordan blocks composing the matrix are either $\left(\begin{array}{cccc}a_{i} & & & \\ 1 & a_{i} & & \\ & \ddots & \ddots & \\ & & 1 & a_{i}\end{array}\right)$ either $\left(\begin{array}{cccc}B & & & \\ I_{2} & B & & \\ & \ddots & \ddots & \\ & & I_{2} & B\end{array}\right)$ with $B=\left[\begin{array}{cc}p & -q \\ q & p\end{array}\right]$ for $\alpha_{i}=p+\mathrm{i} q$. Note that an eigenvalue can be responsible for more than one block. The number of different blocks an eigenvalue $\lambda$ creates is $\operatorname{dim}(\operatorname{ker}(A-\lambda))$. Similarly, let $\delta_{i}=\operatorname{dim}\left(\operatorname{ker}(A-\lambda)^{i}\right), \delta_{i+1}-\delta_{i}$ is the number of blocks of size at least $i+1$. We can hence know the number of blocks of each size and write a Jordan matrix $J$ consisting of blocks in decreasing size order (any order would be fine). This Jordan matrix is similar to the original matrix $A$.

We finally need to compute the similarity matrix $P$ which will be such that $A=$ $P^{-1} J P$. This matrix is obtained by computing the eigenvectors of the matrix $A$ (or $J$ ). 